\title{
Front Matter: Volume 9124
}

, "Front Matter: Volume 9124," Proc. SPIE 9124, Satellite Data Compression, Communications, and Processing X, 912401 (12 June 2014); doi:

$10.1117 / 12.2072315$

SPIE Event: SPIE Sensing Technology + Applications, 2014, Baltimore, MD, United SPIE. States 


\title{
PROCEEDINGS OF SPIE
}

\section{Satellite Data Compression, Communications, and Processing $X$}

\author{
Bormin Huang \\ Chein-I Chang \\ José Fco. López \\ Editors
}

8-9 May 2014

Baltimore, Maryland, United States

Sponsored and Published by

SPIE 
The papers included in this volume were part of the technical conference cited on the cover and title page. Papers were selected and subject to review by the editors and conference program committee. Some conference presentations may not be available for publication. The papers published in these proceedings reflect the work and thoughts of the authors and are published herein as submitted. The publisher is not responsible for the validity of the information or for any outcomes resulting from reliance thereon.

Please use the following format to cite material from this book:

Author(s), "Title of Paper," in Satellite Data Compression, Communications, and Processing $X$, edited by Bormin Huang, Chein-I Chang, José FCo. López, Proceedings of SPIE Vol. 9124 (SPIE, Bellingham, WA, 2014) Article CID Number.

ISSN: 0277-786X

ISBN: 9781628410617

Published by

SPIE

P.O. Box 10, Bellingham, Washington 98227-0010 USA

Telephone +1 3606763290 (Pacific Time) · Fax +1 3606471445

SPIE.org

Copyright (C) 2014, Society of Photo-Optical Instrumentation Engineers.

Copying of material in this book for internal or personal use, or for the internal or personal use of specific clients, beyond the fair use provisions granted by the U.S. Copyright Law is authorized by SPIE subject to payment of copying fees. The Transactional Reporting Service base fee for this volume is $\$ 18.00$ per article (or portion thereof), which should be paid directly to the Copyright Clearance Center (CCC), 222 Rosewood Drive, Danvers, MA 01923. Payment may also be made electronically through CCC Online at copyright.com. Other copying for republication, resale, advertising or promotion, or any form of systematic or multiple reproduction of any material in this book is prohibited except with permission in writing from the publisher. The CCC fee code is 0277-786X/14/\$18.00.

Printed in the United States of America.

Publication of record for individual papers is online in the SPIE Digital Library.

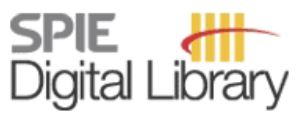

SPIEDigitalLibrary.org

Paper Numbering: Proceedings of SPIE follow an e-First publication model, with papers published first online and then in print and on CD-ROM. Papers are published as they are submitted and meet publication criteria. A unique, consistent, permanent citation identifier (CID) number is assigned to each article at the time of the first publication. Utilization of CIDs allows articles to be fully citable as soon as they are published online, and connects the same identifier to all online, print, and electronic versions of the publication. SPIE uses a six-digit CID article numbering system in which:

- The first four digits correspond to the SPIE volume number.

- The last two digits indicate publication order within the volume using a Base 36 numbering

system employing both numerals and letters. These two-number sets start with 00, 01, 02, 03, 04, $05,06,07,08,09,0 A, 0 B \ldots 0 Z$, followed by 10-1Z, 20-2Z, etc.

The CID Number appears on each page of the manuscript. The complete citation is used on the first page, and an abbreviated version on subsequent pages. Numbers in the index correspond to the last two digits of the six-digit CID Number.

Proc. of SPIE Vol. $9124912401-2$ 


\section{Contents}

ix Conference Committee

\section{SESSION 1 DATA PROCESSING I}

912402 A theory of least-squares target-specified virtual dimensionality in hyperspectral imagery [9124-1]

D. Paylor, C.-I. Chang, Univ. of Maryland, Baltimore County (United States)

912403 Investigation on the GPS single scattering from a 2D largescale sea surface [9124-2]

Y. Wei, L. Guo, Xidian Univ. (China)

912404 Applying region growing algorithm to hyperspectral image for oil segmentation [9124-3] M. Song, X. XU, S. LU, W. XU, H. Bao, Dalian Maritime Univ. (China)

912405 High-resolution remote sensing image restoration based on double-knife-edge method [9124-4]

S. Zhang, L. Wang, X. Shi, X. Wang, X. Shao, Xidian Univ. (China)

912407 Multi-dimensional edge detection operators [9124-6]

S. Youn, C. Lee, Yonsei Univ. (Korea, Republic of)

\section{SESSION 2 DATA COMPRESSION I}

912408 Efficient lossy compression implementations of hyperspectral images: tools, hardware platforms, and comparisons [9124-7]

A. García, L. Santos, S. López, G. M. Callicó, J. F. Lopez, R. Sarmiento, Univ. de Las Palmas de Gran Canaria (Spain)

912409 Lossy hyperspectral image compression using improved classified DCT and 3DSPIHT [9124-8]

K. Wang, Z. Hu, R. Han, J. Zhang, Y. Li, Xidian Univ. (China)

9124 OA Hyperspectral data compression using lasso algorithm for spectral decorrelation [9124-9]

S. A. Alissou, Y. Zhang, Harbin Institute of Technology (China)

$9124 \mathrm{OB} \quad$ Wavelet-based compression of multichannel climate data [9124-10]

E. Sharifahmadian, Y. Choi, S. Latifi, Univ. of Nevada, Las Vegas (United States); S. Dascalu,

F. C. Harris, Univ. of Nevada, Reno (United States) 
9124 OC Anomaly discrimination in hyperspectral imagery [9124-11]

S.-Y. Chen, D. Paylor, C.-I. Chang, Univ. of Maryland, Baltimore County (United States)

9124 OD MTF compensation method utilizing the curved edge for high-resolution satellite image recovery [9124-12]

Q. Luo, L. Wang, H. Yang, S. Zhang, X. Shao, Xidian Univ. (China)

$9124 \mathrm{OE} \quad$ Adaptive sparse signal processing of satellite-based radio frequency (RF) recordings of lightning events [9124-13]

D. I. Moody, D. A. Smith, Los Alamos National Lab. (United States)

$9124 \mathrm{OF} \quad$ A compressed coded aperture imaging warning system [9124-14]

X. Shao, J. Du, L. Wang, Xidian Univ. (China)

9124 OG An adaptive filtering based on generalized sidelobe cancellation for target detection of hyperspectral images [9124-15]

L. Chang, Z.-S. Tang, National Taiwan Ocean Univ. (Taiwan); Y.-L. Chang, National Taipei Univ. of Technology (Taiwan); B. Huang, Univ. of Wisconsin-Madison (United States)

\section{SESSION 4 SPECTRAL UNMIXING}

$9124 \mathrm{OH}$ On the acceleration of the N-FINDER algorithm for hyperspectral endmembers extraction [9124-16]

R. Guerra, S. López, G. M. Callicó, J. F. Lopez, R. Sarmiento, Univ. de Las Palmas de Gran Canaria (Spain)

$9124 \mathrm{Ol} \quad$ Endmember variability resolved by pixel purity index in hyperspectral imagery [9124-17] Y. Li, C. Gao, S.-Y. Chen, C.-I. Chang, Univ. of Maryland, Baltimore County (United States)

$91240 \mathrm{~J}$ On performance improvement of vertex component analysis based endmember extraction from hyperspectral imagery [9124-18]

Q. Du, Mississippi State Univ. (United States); N. Raksuntorn, Suan Sunandha Rajabhat Univ. (Thailand); N. H. Younan, Mississippi State Univ. (United States)

9124 OK Fisher's ratio-based criterion for finding endmembers in hyperspectral imagery [9124-19] C. Gao, S.-Y. Chen, C.-I. Chang, Univ. of Maryland, Baltimore County (United States)

$9124 \mathrm{OL} \quad$ Progressive band processing of simplex growing algorithm for finding endmembers in hyperspectral imagery [9124-20]

R. C. Schultz, M. Hobbs, U.S. Naval Academy (United States) and Univ. of Maryland, Baltimore County (United States); C.-I. Chang, Univ. of Maryland, Baltimore County (United States)

9124 ON Nonlinear hyperspectral unmixing based on constrained multiple kernel NMF [9124-22]

J. Cui, X. Li, Zhejiang Univ. (China); L. Zhao, Hangzhou Dianzi Univ. (China) 
912400 Above the cloud computing orbital services distributed data model [9124-23] J. Straub, Univ. of North Dakota (United States)

9124 OP Parallelized physical optics computations for the RCS prediction of rough surface by CUDA [9124-24]

X. Meng, L. Guo, Xidian Univ. (China)

$9124 \mathrm{OQ}$ Optimizing Weather and Research Forecast (WRF) Thompson cloud microphysics on Intel Many Integrated Core (MIC) [9124-25]

J. Mielikainen, B. Huang, A. Huang, Univ. of Wisconsin-Madison (United States)

9124 OR Computational design of miniaturized microstrip antenna for satellite communications in the $S$ and $C$ bands [9124-26]

J. I. Marulanda Bernal, D. A. Campo Caicedo, Univ. EAFIT (Colombia)

9124 OS Massive parallel implementation of JPEG2000 decoding algorithm with multi-GPUs [9124-27]

X. Wu, Y. Li, K. Liu, K. Wang, L. Wang, Xidian Univ. (China)

9124 OT Using Intel Xeon Phi to accelerate the WRF TEMF planetary boundary layer scheme [9124-28]

J. Mielikainen, B. Huang, A. Huang, Univ. of Wisconsin-Madison (United States)

\section{SESSION 6 DATA COMPRESSION II}

9124 OW Lossless compression of hyperspectral images using C-DPCM-APL with reference bands selection [9124-31]

K. Wang, H. Liao, Y. Li, S. Zhang, X. Wu, Xidian Univ. (China)

9124 OX Remote sensing image progressive transmission based on socket with retry broken downloads [9124-32]

H. QU, Liaoning Technical Univ. (China) and Harbin Institute of Technology (China);

Y. Meng, W. Liu, X. Shan, J. Yu, Liaoning Technical Univ. (China)

\section{SESSION 7 IMAGE CLASSIFICATION}

9124 OY Land cover classification in multispectral satellite imagery using sparse approximations on learned dictionaries [9124-33]

D. I. Moody, S. P. Brumby, J. C. Rowland, G. L. Altmann, Los Alamos National Lab. (United States)

$9124 \mathrm{OZ}$ Sparse classification of hyperspectral image based on first-order neighborhood system weighted constraint [9124-34]

J. Liu, Xidian Univ. (China); H. Guan, Beijing Institute of Spacecraft System Engineering (China); J. Li, Y. Li, Xidian Univ. (China) 
912410 An efficient spatial-spectral classification method for hyperspectral imagery [9124-35] W. Li, Beijing Univ. of Chemical Technology (China); Q. Du, Mississippi State Univ. (United States)

$912411 \quad$ A stereo remote sensing feature selection method based on artificial bee colony algorithm [9124-36]

Y. Yan, P. Liu, Y. Zhang, N. Su, S. Tian, Harbin Institute of Technology (China); F. Gao,

Heilongjiang Academy of Sciences (China); Y. Shen, Harbin Institute of Technology (China)

912413 Background suppression issues in anomaly detection for hyperspectral imagery [9124-38] Y. Wang, Univ. of Maryland, Baltimore County (United States) and Harbin Engineering Univ. (China); S. Chen, Harbin Engineering Univ. (China); C. Liu, China Agricultural Univ. (China); C.-I. Chang, Harbin Engineering Univ. (China)

$912414 \quad$ No-reference remote sensing image quality assessment using a comprehensive evaluation factor [9124-39]

L. Wang, X. Wang, X. Li, X. Shao, Xidian Univ. (China)

912415 Impact of a revised standard for best practices for academic, governmental and industrial ground station scheduling and communications design [9124-40]

S. D. Kerlin, J. Straub, C. Korvald, Univ. of North Dakota (United States)

912418 Manifold regularized sparsity model for hyperspectral target detection [9124-43]

J. Li, X. Li, Zhejiang Univ. (China); L. Zhao, Hangzhou Dianzi Univ. (China)

POSTER SESSION

912419 A novel IR polarization imaging system designed by a four-camera array [9124-44]

F. Liu, X. Shao, P. Han, Xidian Univ. (China)

$91241 \mathrm{~A} \quad$ Imaging characteristics of ball lens [9124-45]

Q. Li, X. Shao, Xidian Univ. (China)

9124 1B Spherical aberration and modulation transfer function [9124-46]

Q. Li, X. Shao, Xidian Univ. (China)

9124 1C Focusing through a turbid medium by amplitude modulation with genetic algorithm [9124-47]

W. Dai, L. Peng, X. Shao, Xidian Univ. (China)

9124 ID Particle swarm optimization for focusing by phase modulation through scattering media [9124-48]

L. Peng, W. Dai, X. Shao, Xidian Univ. (China)

9124 IE Pixel-level image reconstruction method of polarization images acquired by multiaperture imaging systems [9124-49]

P. Han, F. Liu, X. Shao, Xidian Univ. (China) 
$9124 \mathrm{IF}$ Online visual tracking based on updating with smoothing [9124-50] J. Zhang, K. Liu, F. Cheng, Y. Li, Xidian Univ. (China)

9124 IG Random grid fern for visual tracking [9124-51]

F. Cheng, K. LiU, J. Zhang, Y. Li, Xidian Univ. (China)

Author Index 


\title{
Conference Committee
}

\author{
Symposium Chair
}

David A. Whelan, Boeing Defense, Space, and Security

(United States)

Symposium Co-chair

Wolfgang Schade, Technische Universität Clausthal (Germany) and

Fraunhofer Heinrich-Hertz-Institut (Germany)

Conference Chairs

Bormin Huang, University of Wisconsin-Madison (United States)

Chein-I Chang, University of Maryland, Baltimore County (United States)

José Fco. López, Universidad de Las Palmas de Gran Canaria (Spain)

Conference Co-chairs

Chulhee Lee, Yonsei University (Korea, Republic of)

Yunsong Li, Xidian University (China)

Qian Du, Mississippi State University (United States)

Conference Program Committee

Philip E. Ardanuy, Raytheon Intelligence \& Information Systems (United States)

Roberto Camarero, Centre National d'Études Spatiales (France)

Lena Chang, National Taiwan Ocean University (Taiwan)

Ni-Bin Chang, University of Central Florida (United States)

Yang-Lang Chang, National Taipei University of Technology (Taiwan)

David J. Crain, GeoMetWatch Corporation (United States)

Mitchell D. Goldberg, National Oceanic and Atmospheric

Administration (United States)

Lingjia Gu, Jilin University (China)

Li-xin Guo, Xidian University (China)

Tung-Ju Hsieh, National Taipei University of Technology (Taiwan)

Allen H.-L. Huang, University of Wisconsin-Madison (United States)

Felix Huber, Deutsches Zentrum für Luft- und Raumfahrt e.V. (Germany)

Roger L. King, Mississippi State University (United States)

Sebastian Lopez Suarez, Universidad de Las Palmas de Gran Canaria (Spain) 
Enrico Magli, Politecnico di Torino (Italy)

Jarno Mielikainen, University of Eastern Finland (United States)

Daniela I. Moody, Los Alamos National Laboratory (United States)

Antonio J. Plaza, Universidad de Extremadura (Spain)

Jordi Portell de Mora, Universidad de Barcelona (Spain)

Jeffery J. Puschell, Raytheon Space \& Airborne Systems

(United States)

Shen-En Qian, Canadian Space Agency (Canada)

Joan Serra-Sagrista, Universidad Autònoma de Barcelona (Spain)

Xiaopeng Shao, Xidian University (China)

Carole Thiebaut, Centre National d'Études Spatiales (France)

Pierre V. Villeneuve, Space Computer Corporation (United States)

Raffaele Vitulli, European Space Research and Technology Centre (Netherlands)

Maria F. von Schoenermark, Universität Stuttgart (Germany)

Jiaji Wu, Xidian University (China)

Zhensen Wu, Xidian University (China)

Zhang Ye, Harbin Institute of Technology (China)

Session Chairs

1 Data Processing I

Bormin Huang, University of Wisconsin-Madison (United States)

2 Data Compression I

Chein-I Chang, University of Maryland, Baltimore County

(United States)

3 Data Processing II

José Fco. López, Universidad de Las Palmas de Gran Canaria (Spain)

4 Spectral Unmixing

Chulhee Lee, Yonsei University (Korea, Republic of)

$5 \quad$ High-Performance Computing

Yunsong Li, Xidian University (China)

6 Data Compression II

Qian Du, Mississippi State University (United States)

7 Image Classification

Daniela I. Moody, Los Alamos National Laboratory (United States)

8 Data Processing III

Meiping Song, Dalian Maritime University (China) 